\title{
Ethik im medizinischen Alltag - eine amerikanische Meinung
}

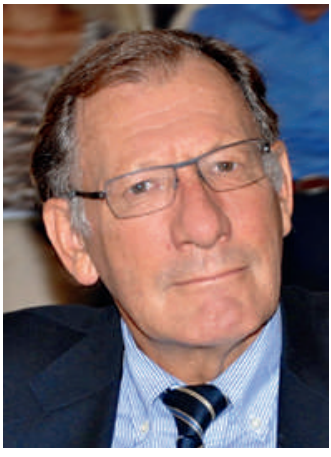

Jean Martin
Die Erfahrung des Arztes im Bereich Ethik hängt von seiner Ausbildung und seiner Praxis ab (von deren Geographie und Sozio-Anthropologie, wenn man so will). Natürlich auch von seinem persönlichen Standpunkt, wobei dieser allerdings nicht stärker ins Gewicht fallen sollte als die Werte der Patienten selbst. In einer jüngsten Ausgabe des Hastings Center Report [1] stiess ich auf einen Text von William T. Branch, vormals Leiter der Allgemeinen Inneren Medizin der Universität Emory in Atlanta, einem der grossen akademischen Zentren in den USA. Er lehrt auch «Medical humanities».

Branch beschreibt seine berufliche und persönliche Entwicklung: «Ich war in den 1960er Jahren zunächst Medizinstudent und später Assistenzarzt. In jener Zeit hatte die Medizin als 〈Glaubenssystem〉 gerade einen Höhepunkt erreicht. Der Rahmen dafür war gesteckt durch verschiedene «Wunder», darunter die Entdeckung der Antibiotika und anderer Medikamente in den 30er und 40er Jahren. Meine Generation befasste sich mit Medizin im absoluten Glauben an die Objektivität der Wissenschaft. Unsere Lehrmeister arbeiteten in Labors und sprachen von der absoluten Hingabe an die Arbeit und von Aufopferung.»

Wissenschaft und/oder sinnloser Aktionismus - Nicht leiden lassen. «Erschöpft durch den Mangel an Schlaf und bestimmte «futile» Praktiken - diese Qualifizierung wurde damals jedoch kaum verwendet - kämpften meine Kollegen und ich bis zum Letzten, um Leben von unheilbar kranken und in der Endphase befindlichen Patienten zu retten. Diese Kranken hatten enorm darunter zu leiden, und ich konnte einfach das idealistische Bild, das ich von mir selbst hatte, nicht mit der makabren Realität vereinbaren: ein emphysematischer Patient, den ich intubiert und fünf Mal auf die Intensivstation verlegt hatte, bevor er dann schliesslich verstarb; eine halb komatöse Frau nach der Chemo, ohne Überlebenschance, die sich wehrte, während ich nach einer Vene suchte; ein Mann voller Metastasen im Brustkorb, den ich heftig pumpte. Diese Erinnerungen habe ich fast ein Jahrzehnt lang verdrängt. Ich konnte nicht darüber nachdenken, bis ich eine humanistischere Seite der Medizin gelebt habe.»

«Erst als wir in den 70ern in einer Sackgasse gelandet waren, begannen die Ärzte, sich Fragen zu stellen. Durch die Verbesserungen bei den Beatmungsgeräten und der Intensivpflege konnten im Endstadium befindliche Patienten noch Wochen und Monate in halb-bewusstem oder komatösem Zustand am Leben erhalten und damit ihre Existenz auf eine Art und Weise verlängert werden, die zu nichts führte (pointless). Diese für die Patienten unwürdigen Praktiken und die Verschlimmerung ihres Leidens waren eklatant.» Seither - so Branch - haben Kommunikationsfähigkeiten, Bioethik und später auch die Palliativpflege Einzug in die medizinische Ausbildung gehalten.

Autonomie des Patienten - er kann selbst über seine Würde entscheiden. «Dass ich sehen musste, wie Patienten ihre Autonomie, ihre Kontrolle und letztlich auch sich selbst (loss of the self) verloren haben, hat meine Einstellung verändert. Wir können Schmerzen behandeln, aber wir können nicht die mit fortschreitender Krankheit erfolgende Zerstörung der Persönlichkeit rückgängig machen. Wer identitätsbedrohendes Leid erfahren - und überwunden - hat, kann bezeugen, dass dies Horror ist».

Bedeutung von Leid - gibt es gutes Leid? «Einige Weise glauben, dass Leid eine spirituelle Bedeutung hat. Ich glaube, dass wir als Ärzte unsere Ethikregeln für den Menschen und innerhalb menschlicher Werte geltend machen müssen. Wir müssen an der Seite unserer Kranken stehen und uns ihrem Leid widersetzen. Wir müssen das verteidigen, was ihnen gut tut.»

Wenn der Patient frei entscheidet, auf eine bestimmte Weise zu leben, ist es sicherlich nicht an uns, ihm die Freiheit zu verweigern, grosses Leid ohne optimale Schmerzbehandlung zu ertragen. Gleichzeitig sollte auch nicht verneint werden, dass dies - sofern es sich um eine wohlüberlegte Entscheidung des Betroffenen handelt - verdienstvoll sein kann. Es kann jedoch nicht mehr angehen, dass die Pflegenden dem Kranken eine solche Sicht möglicherweise aufzwingen, indem er durch Unterlassung oder, noch schlimmer, durch explizite Ablehnung ausreichender Schmerztherapien ‘zum Leiden genötigt` wird. An dieser Stelle gilt es an einen Satz zu erinnern, der überraschen mag: «In der Pflege gilt zuallererst die Moral des Patienten, nicht die des Pflegenden.»

In der Schweiz. War die Situation («wissenschaftliche Objektivität, die den Menschen verbirgt» Branch) dem vergleichbar, was von den Vereinigten Staaten gesagt wird? Möglicherweise war dort der «nicht diskutierbare Glaube an die Macht der Medizin, der zu Praktiken führte, die den Menschen und seine Bedürfnisse nicht ausreichend berücksichtigten», ausgeprägter als bei uns. Aber die Tendenzen waren dieselben. Und viele könnten mit Branch sagen: «Im Umgang mit meinen Kranken habe ich im Praxisalltag die Erfahrung gemacht, dass sich wissenschaftliche Arroganz und Stolz (hybris) zu einer eher mitgefühlsgeprägten Einstellung wandelten.»

Jean Martin, Mitglied der Redaktion 